\title{
Pattern of Animal Bites and Post Exposure Prophylaxis for Rabies: An One Year Study in Bangladesh Institute of Tropical Infectious Disease (BITID) Chattogram
}

\author{
Md Mamunur Rashid ${ }^{1 *}$ \\ M A Hassan Chowdhury ${ }^{1}$ \\ Md Hossain Rashid Chowdhury ${ }^{1}$
}

'Department of Clinical Tropical Medicine Bangladesh Institute of Tropical Infectious Disease (BITID) Chattogram, Bangladesh.

\footnotetext{
${ }^{*}$ Correspondence to:

Dr. Md Mamunur Rashid Associate Professor

Department of Clinical Tropical Medicine Bangladesh Institute of Tropical Infectious Disease (BITID) Chattogram, Bangladesh. Mobile : +8801711236644

Email:mamunurdr30@gmail.com
}

Date of Submission : 19.11 .2020

Date of Acceptance ： 26.12 .2020

www.banglajol.info/index.php/CMOSHMCJ

\begin{abstract}
Background: Rabies is a global disease occurring in more then 150 countries including Bangladesh. It was neglected in Bangladesh before 2010 with more than 2000 annual rabies case. Number of rabies patients are declining in whole world due to more availability of resources needed for rabies prevention. In this background here our objective is to study the Post Exposure Prophylaxis (PEP) in rabies \& to observe the pattern of animal bites in Bangladesh Institute of Tropical and Infectious Disease (BITID) which is a national institute dealing Tropical \& Infectious disease of Bangladesh.
\end{abstract}

Materials and methods: It was a retrospective study carried out among bite patients coming to BITID (Both outdoor \& indoor) from 01.01.2017 to 31.12.2017 where a registry of all post exposure treatment was maintained. Total 1863 cases of animal bite patients came to BITID over the study period. The data were analyzed finally.

Results: Among 1836 cases, 1185(63.66\%) were male and 678 (34.44\%) were female. 1155 cases (62\%) belonged to $>12$ years of age and 708 (38\%) cases belonged $\leq 12$ years of age 815(43.74\%) cases were category III bite. 1386 (74.4\%) cases were bitten by dog \& bites in 1110 (59.6\%) cases occurred in lower limbs. Most patients 1138 cases (61.1\%) came from rural areas \& maximum bites occurred from March 2017 to September 2017.

Conclusion: Animal bite is a great burden for Bangladesh. To prevent it we need public awareness, mass dog vaccination, street dog control and complete post exposure prophylaxis as per schedule given by national rabies elimination program of Bangladesh government.

Key words: $\mathrm{PEP}=$ Post Exposure Prophylaxis; $\mathrm{ARV}=$ Anti Rabies Vaccine; ERIG= Equine Rabies Immunoglobulin; PVRV= Purified Verocell Rabies Vaccine; PCEC= Purified Chick Embryo Cell Caccine; FAT= Fluorescent Antibody Test.

\section{INTRODUCTION}

Rabies is a global disease occurring in more than 150 countries and territories ${ }^{1}$. The infection prevails among domestic \& wild animals, of them dogs are the main source of human rabies transmitted by close contact with infectious material, usually saliva, bites, or scratches. Other rabid animals are cat, monkey fox, jackals, bats \& other wild animals. Dogs are the source of infection in all human rabies deaths in Asia and Africa whilst bats are the source of most human rabies death in America, Australia \& Western Europe. Human deaths following exposure to foxes, jackals , raccoons and other wild carnivore host species are very rare.

The exposure to animals can be categorized by WHO as Category I, Category II, Category III bites ${ }^{2}$. Category I =touching or feeding animals, licks on the skin. Category II =nibbling of uncovered skin, minor scratches or abrasions without bleeding, licks on broken skin. Category III =single or multiple transdermal bites or scratches, contamination of mucous membrane with saliva from licks. Before onset of sign \& symptoms of rabies, no test is available to diagnose it. The reference method for diagnosing rabies is the Fluorescent Antibody Test (FAT) recommended by WHO. 
The diagnosis can be mode by detecting whole virus, viral specific antibodies in the CSF or nucleic acid in infected tissues (Brain, skin, urine or saliva) ${ }^{3}$. Rabies can be reliably diagnosed from brain samples taken after death. Cerebral inclusion bodies (Negri bodies) are 100\% diagnostic for rabies infection but are found in only about $80 \%$ cases $^{4}$. Though rabies is $100 \%$ fatal, effective treatment soon after exposure to rabies can prevent the onset of disease. This is known as post exposure prophylaxis ${ }^{5}$. According to the report of world Health organization (WHO) more than 15 million people are treated for rabies due to animal bite in order to prevent rabies in different point of the world annually ${ }^{6}$. Also around 60,000 cases of human deaths resulting from rabies are reported in worldwide annually ${ }^{7}$. Rabies has existed in Bangladesh for a long time, so that the disease is endemic in our country.

It claims more than 2000 lives annually in Bangladesh ${ }^{8}$. Annual number of dog bite in Bangladesh varies between 200,000 and 300,000 and $95 \%$ of rabies death occurs due to rabid dog bites $^{9}$. Our study was performed to identify the current pattern of animal bites and to evaluate postexposure prophlaxis at BITID, Fauzdarhat, Chattogram, Bangladesh. BITID is providing rabies post exposure prophylaxis among animal bite patients. Since September 2014 it had been made a center for vaccination by CDC of the DGHS of the Government of Bangladesh. This institute is situated at Fouzderhat under Sitakund Upazila of Chattogram. It is a newly built national institute raised over the existing infectious diseases hospital of Bangladesh. Animal bite patients from all over the city and also from the near by Upazila of Chattogram come to BITID for post exposure prophylaxis and advices. The hospital has dedicated team of trained physicians, nurses \& other personal to manage effectively the victims of animal exposure according to national guidelines of rabies elimination programme of Bangladesh Government under Communicable Disease Control (CDC), Director General of Health Services (DGHS).

\section{MATERIALS AND METHODS}

It was a retrospective study conducted from January to December 2017. The study was aimed to identify pattern of animal bites and post exposure prophylaxis for rabies. The basic details of all animal exposure like name, age, sex residence, contact number, location of bite, category of bite, animals involved, care given to wound \& details of PEP was recorded in a register supplied by CDC, DGHS. PEP was started without delay according to WHO criteria, eg category III bites were given wash plus ARV plus ERIG and category II were give wash \& ARV. No treatment was given in category I patients. A card was supplied to every patient containing all details about PEP.

Every patient was asked individually to report to the center for any thing abnormal they notice following the prophylaxis. Patients who were severely injured got admission to BITID \& got PEP \& other treatment. The patients had been contacted individually 2 to 6 months following vaccinations to know their status. The wound area is cleaned with simple soap and running tap water for 20 minutes to wash the virus and also concomitant bacteria. Usually suturing of wound area is not done unless profuse bleeding where less number of suturing can be done after rabies immunoglobulin administration in and around the wound. For PEP we used two kinds of inactivated anti rabies cell culture vaccines normally Purified Vero Cell Rabies Vaccine (PVRV) and Purified Chick Embryo Cell Vaccine (PCEC) and most of them were supplied by government of Bangladesh. Cold chain was maintained \& intradermal route was followed on day $0,3,7 \& 28$ days at dose $0.1 \mathrm{ml}$ on both upper arm. Immunoglobulin's used were Equine source of Rabies Immunoglobulin (ERIG) at a dose of $40 \mathrm{iu} / \mathrm{kg}$ body weight in and around the wound. All data's of year 2017 from registries were extracted after obtaining permission from the hospital authority. After compiling all the data we had analyzed them and made the inference.

\section{RESULTS}

There were total 1863 case of animal exposure presented to BITID over the study period. All of them were analyzed.

Table I : Age distribution among animal bite patients $(\mathrm{n}=1863)$.

$\begin{array}{lrr}\text { Age } & \text { Number } & \text { Percent }(\%) \\ \leq 12 \text { years } & 708 & 38 \% \\ >12 \text { years } & 1155 & 62 \% \\ \text { Total } & 1863 & 100 \%\end{array}$

Vulnerable age group was more than 12 years.

Table II : Sex distribution among animalbite patients $(\mathrm{n}=1863)$

\begin{tabular}{lrr} 
Sex & Number & Percent (\%) \\
Male & 1185 & $63.66 \%$ \\
Female & 678 & $34.44 \%$ \\
Total & 1863 & $100 \%$ \\
\hline
\end{tabular}

Among the study subjects males were predominately affected $(63.66 \%)$. Male female ratio were 1.7:1.

Table III : Regional distribution of patients $(n=1863)$.

$\begin{array}{lrr}\text { Area } & \text { Number } & \text { Percent (\%) } \\ \text { Urban } & 725 & 38.9 \% \\ \text { Rural } & 1138 & 61.1 \% \\ \text { Total } & 1863 & 100 \%\end{array}$

Most of the patients came from rural area $61.1 \%$.

Table IV : Body site distribution among animal bite patients $(n=1863)$.

\begin{tabular}{lrr} 
Site & Number & Percent (\%) \\
Lower limb & 1110 & $59.6 \%$ \\
Upper limb & 394 & $21.1 \%$ \\
Trunk & 158 & $8.48 \%$ \\
Head \& neck & 143 & $7.67 \%$ \\
Multiple site & 58 & $3.1 \%$ \\
Total & 1863 & $100 \%$ \\
\hline
\end{tabular}

Lower limbs were predominately affected (59.6\%). 
Table $\mathbf{V}$ : Types of bitting animals involved $(\mathrm{n}=1863)$.

\begin{tabular}{lrr} 
Types of Animals & Number & Percent (\%) \\
Dogs & 1386 & $74.4 \%$ \\
Others & 477 & $25.6 \%$ \\
Total & 1863 & $100 \%$ \\
\hline
\end{tabular}

Dog were the commonest animal.

Table VI : Category of bite $(\mathrm{n}=1863)$.

\begin{tabular}{lrr} 
Category of bite & Number & Percent (\%) \\
Category I & 296 & $15.88 \%$ \\
Category II & 752 & $40.36 \%$ \\
Category III & 815 & $43.74 \%$ \\
Total & 1863 & $100 \%$ \\
\hline
\end{tabular}

Category III was predominant (43.74\%).

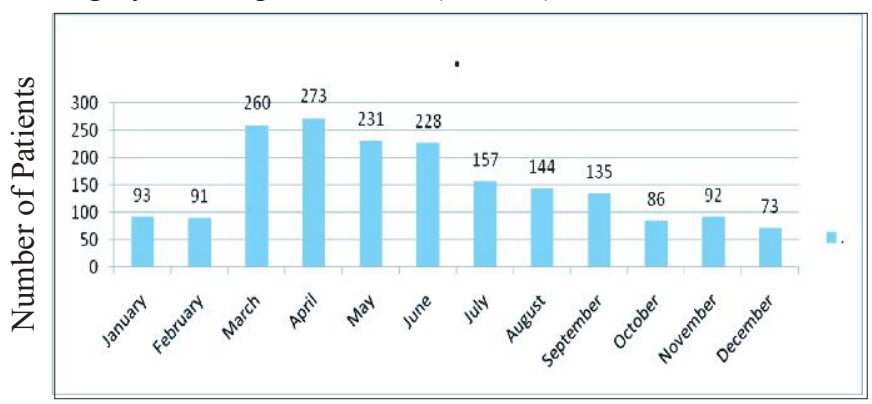

Months of the year 2017

Figure 1 : Month wise distribution of animal bite patients $(n=1863)$.

\section{DISCUSSION}

We found that large number of animal bite presented in a single tertiary care hospital in Bangladesh for post exposure treatment against rabies. Extrapolation of these figures may help to judge approximate national burden in terms of animal exposures. Results of the study showed most of the patients are more than 12 years of age. In BITID>12 years old child's are considered as adult. So, Adults are more affected then child in this study. Though vulnerable age of animal bite is child but in our instuition, the scenerio is different.

About $64 \%$ of bitten patients were male which is consistent with previous studies done in other countries ${ }^{10}$. The reason of higher occurrence of animal bites in male patients is probably that they spend more time in outdoor then women. Most of the patients affected were from rural areas. This finding may be due to increase in travelling people in rural and agricultural areas as well as increased activity of animals seeking foods.
Considering anatomic location of the bites, the majority of cases were related to lower limb (60\%). which is consistent with other studies ${ }^{11}$. The predominant bite site in upper limb may be due to that here most patients are adult $\&$ lower limb is near to level of dog height. Our cases were predominantly victims of dog bite which is consistent with others study's where most of the bites were by $\operatorname{dog}^{12.13}$. Moore et al reported also that the incidence of dog bite was $75 \%{ }^{14}$. Highest category patients were category III. Though animal bites are reported to be more common in September-October month, but in out institution we found highest incidences were in April followed by March.

\section{LIMITATIONS}

As we used only data's which were previously included in the government register of CDC, DGHS, Bangladesh, we were unable to collect some other variables like socio-economic status, education level, provocation status. However our study was a limited study that was conducted in BITID only.

\section{CONCLUSION}

Nationwide CDC is the executive body governing rabies elimination in Bangladesh and BITID is a control and prevention center regarding this under the supervision of CDC. Among the prophylaxis provided at BITID maximum animals bites were found $\operatorname{dog}$ bite $\&$ most of them were category III bite \& most common sites were lower limb. Patients with more severe bites came earlier than the lesser bites in our center. Public awareness should be increased by mass media to come to health center as soon as possible after bite

Health care providers should be well trained in this regard \& also health policy-makers should ensure rabies control within animal populations and appropriate supplies of post exposure rabies treatment and antibiotic prophylaxis for bitten people. Complete course of PEP as per schedule of national rabies elimination program of Bangladesh government must be ensured. Continued surveillance and rabies control along with mass dog vaccination is still necessary.

\section{DISCLOSURE}

All the authors declared no competing interest. 


\section{REFERENCES}

1. Fevre EM, Kaboyo RW, person V, Edelsten M, Coleman PG,Cleaveland S. The epidemiology of animal bite injuries in Uganda and projections of the burden of rabies. Trop Med Int. Health.2005:10(8):790-798.

2. $\quad$ WHO expert Consultation on Rabies, Second report. WHO Technical report series 982.

3. Senanayake Abeysinghe Mvdiyanselage Knlaratne, Koasala Weerakoon, UshaKumari Bokalamulla, Nanada Abagasoitiya. Pattern of animal bites and post exposure prophylaxis in rabies: A five year study in a tertiary care unit in Srilanka.BMC Infection Diseases BMC Series.2016;16:62.

4. $\quad$ Rabies (Part I). WER Srilanka. 2014;41(38):1-4.

5. $\quad$ Rabies (Part II). WER Srilanka. 2014;41(39):1-4.

6. World Health Organization. Human Rabies. Geneva:WHO. 2013.

7. World Health Organization. Human Rabies. Geneva: WHO. 2016.

8. Health Bulletin. Government of the peoples Republic of Bangladesh, Ministry of Health and Family Welfare. 2015.

9. WHO.WHO expert consultation on Rabies.Firstreport:WHO technical report series.2004;931:1-121

10. Khajaei S, Rezaeian S, Soheylizad M, Gholamaliee B. Factors associate with delay in post exposure prophylaxis in bitten people. Medical Journal of the Islamic Republic of Iran. 2014; 28:158.

11. Tenzin, DhandNK, Gyeltshen T, Firestone S, Zangmo C, Dema C et al. Dog bite in human and estimating human rabies mortality in rabies endemic areas of Bhutan. PLOSNegl Trop Dis. 2011;5(11):e1391.

12. Khazaei S, Rezaeian S, Salehiniya H, Reazaei R, SabzavariJTN, Sohelyazad M. Dealy in post exposure prophylaxis and associated factor among people bitten by animals in the north east of Iran, 2015. Archives of clinical Infectious Diseases. 2016

13. Poorolajal J, Bahaee I, Yoosefi R, Farnoogh F, Animal bite \& deficiences in Rabies post exposure prophylaxis in Tehran, Iran, Arch Iran Med. 2015; 18(12): 822-826

14. Moore DA, SischoWM, Hunter A, Ariles T. Animal bite epidemiology and surveillance for rabies exposure prophylaxis. JAVMA 2000;2017(2):190-194 